\title{
Research on the Content of "Explore the Regular Patterns" in Elementary Textbooks
}

\author{
Yan-chun Zhang \\ School of Mathematics and Information, LeShan Normal University, Leshan, Sichuan, China \\ Zhangyanchun201@163.com
}

Keywords: Explore Regular Patterns, Teaching Value, Teaching Material Research, Teaching Suggestion

\begin{abstract}
The content of "explore the regular patterns" in primary school mathematics has unique teaching value, and teachers' teaching should be based on the overall grasp of curriculum standard and teaching material.. The paper takes the version of Southwest Normal University Press as an example to show the following features: compiling with unit form and dispersed form; the unit has unified presentation form; the patterns have diversified explicit types and with open-ended questions. It is recommended that teachers first grasp the characteristics of textbook compilation, understand the intention of compiling textbooks, and creatively use textbooks on the basis of understanding the requirements of standards. Secondly, in teaching design and implementation, we should leave enough room for students to explore, emphasize process evaluation, and pay attention to students' learning process and emotional attitude.
\end{abstract}

\section{小学数学“探索规律”内容的教学价值及教材研究}

\author{
张彦春 \\ 数学与信息学院, 乐山师范学院, 乐山, 四川, 中国 \\ Zhangyanchun201@163.com
}

关键词: 小学数学, 探索规律, 教材研究; 教学建议

摘要：小学数学教材中的“探索规律”内容具有独特的教学价值, 教师教学要基于课标和教材的 整体把握。研究发现小学数学教材“探索规律”内容编写采用了单元集中编写与穿插编写相结的 形式, 设计了多种形式的探索规律问题, 同时注意了问题的开放性等特点。建议教师在教学中 要结合《标准》要求，把握教材的编写意图和特点，创造性的使用教材; 在教学设计以及实施 中要给学生留下足够的探索空间, 突出过程性的学习评价, 关注学生学习过程和情感态度价值 观教学目标的实现。

\section{1. 引言}

“探索规律”是数学学习和问题解决过程中的一种常见活动。《义务教育数学课程标准》将 “探索规律”列为数与代数领域的独立学习内容, 它的地位、教学价值以及实施方式的研究已经 引起人们的关注[1-3]。在中小学数学教学中, 教师对“探索规律”内容本身以及它在现行教材中 的整体编排，包括具体内容、呈现方式、问题背景等还缺乏较为系统的认识和理解，导致在教 学设计和教学实施中不能站在新课标以及教材整体的高度来把握,难以实现其教学价值。基于 课程标准基本要求，以西南师范大学出版的小学数学教材 (简称“西师版小学数学教材”) 为研 究对象对此问题展开研究，以期对该部分内容的教学实践提供一定的参考。 


\section{2. 小学数学“探索规律”内容的价值}

\section{1 对“探索规律”的认识}

“探索”一般是指“多方寻求答案，解决疑问”，在自然语境中，“探索”常与“发现”、“问题”、 “科学”联系在一起。《义务教育数学课程标准(2011版)》[4] (以下简称《标准》) 对“探索”的界 定是指“独立或与他人合作参与特定的数学活动, 理解或提出问题, 寻求解决问题的思路, 发 现对象的特征及其与相关对象的区别和联系，获得一定的理性认识”。“规律”一般是指“事物之 间的内在的本质联系和发展趋势”,是揭示事物之间或现象之间“关系”的一种模型 [2]。在义务教 育阶段, 数学研究的主要对象是“数”和“形”, 在一个过程中, 一组“数”或“形”反复出现（或按 照某种必然趋势出现），这一组数或形构成一个“模式”（pattern），模式中单元之间稳定的、 本质的联系就是“规律”（rule）。因此，小学数学中“探索规律”可理解为独立或与他人合作， 对客观事物和现象之间内在、稳定、反复出现的关系进行认识的过程。这一过程首先是要明确 构成规律的元素以及依次不断重复出现的基本单元（了解规律结构）, 然后还需要能根据规律 的重复特性预测出后面的元素（预测发展趋向）[2]。探索规律内容的学习有利于“培养学生数 感、符号感”, “培养学生观察能力, 推理能力, 抽象思维能力”, 同时也“是培养学生发散性思 维, 渗透数学建模思想等的良好载体”。

\section{2 “探索规律”内容教学要求与基本定位}

关于“探索规律”, 《标准》对第一学段的内容要求是“探索简单情境下变化规律”, 第二学 段的内容要求是“探求给定情境中隐含的规律或变化趋势”, 并给出四个例题对此加以说明。结 合《标准》要求和现有教材中的相关实例, 我们认为“探索规律”内容在小学数学教材中有如下 定位。首先，从内容的层次上来讲，“规律”的学习不应将其视作为是教材中相应具体知识的“拓 展”和“深化”, 它与知识、技能的学习不是一种“依附”关系, 而应将其视作为与教材中“知识” 平行，甚至略高的一种内容地位。其次，《标准》中“规律”的理解不能“泛化”，它是指“变化 规律”、“变化趋势”以及“给定情境中隐含的规律”, 前者强调“变化情境中的”, 能依据所呈现的 对象形式写出下一个对象，后者应是指特定的实例、现象或一组材料中隐含的数量规律。

\section{3. 小学数学“探索规律”内容教材研究}

新课程实施后, 国内使用的各种小学数学新教材都按照《标准》的要求, 编写了各种形式 的“探索规律”内容。如人教版通过“数学广角”、“找规律”两种栏目形式编写独立单元设计的“探 索规律” 内容, 内容包括搭配中的数学规律, 植树中的数学规律, 图形和数的变化规律等。苏 教版在四年级和五年级集中编写了五个单元的“找规律”内容，内容涉及了间隔排列、周期现象、 框数中的数学规律等。为了较为具体、深入的研究小学数学教材中“探索规律”内容, 以下仅以 西南师范大学出版社编写的《义务教育课程标准实验教科书 (小学数学)》 [5] (以下简称西师 版小学数学教材) 为例, 对小学数学教材中“探索规律”的内容的编写特征, 价值取向以及教学 进行研究。

\section{1 编写方式上采用单元集中和穿插渗透相结合}

纵观西师版小学数学教材, “探索规律”内容的编写采用了独立单元编写和渗透、穿插编写 并重的模式。教材在二（下）、三（上）、三（下）、四（上）、四（下）以独立节的形式编写了 六个“探索规律”的单元, 如表 1 所示。这六个主题在认知和思维要求上层层递进, 低段主要以 感知、描述重复性出现, 递增、递减或简单倍数规律为主, 高段主要以探索关系性规律为主。 这些以独立单元形式编写的“探索规律”内容非常注重问题背景与现实生活、自然现象相联系, 例如在二 (下) 教材中设计了“生活中的数学规律”, 通过向学生呈现斑马花纹, 花瓣数量, 丝 织品花色让学生感知生活中的数学规律, 初步让学生认识到数学与生活、自然的联系。除了以 单元集中的形式编写外，在一年级到六年级的每一册中，教材还结合该册的具体内容，将“探 索规律”的六个主题通过例题、课堂活动、练习题、思考题形式进行穿插渗透编写。进一步统 
计后发现教材中集中编写和穿插编写的探索规律问题在数量非常接近，如图 1 所示。这表明探 索规律内容的教学我们应遵循循序渐进, 螺旋上升的方式, 不应该毕其功于一役, 急于求成。 特别是对于穿插渗透编写的这一部分内容教学, 我们在教学时仍应该突出“探索规律”内容的本 质, 让学生经历探索规律的过程, 来培养学生的观察、比较、分析、猜想、验证等思维能力。

表 1 西师版小数教材中“探索规律”单元统计

\begin{tabular}{cccc}
\hline 年级 & 二 & 三 & 四 \\
\hline 上册 & & 数的倍数规律 & 利用计算器探索特殊数的 \\
& & & 运算规律 \\
下册 & $\begin{array}{c}\text { 图形、符号排列规律; } \\
\text { 生活中的数学规律 }\end{array}$ & 图表中数的排列规律 & 数与形结合的数量关系规 \\
& & 律 \\
\hline
\end{tabular}

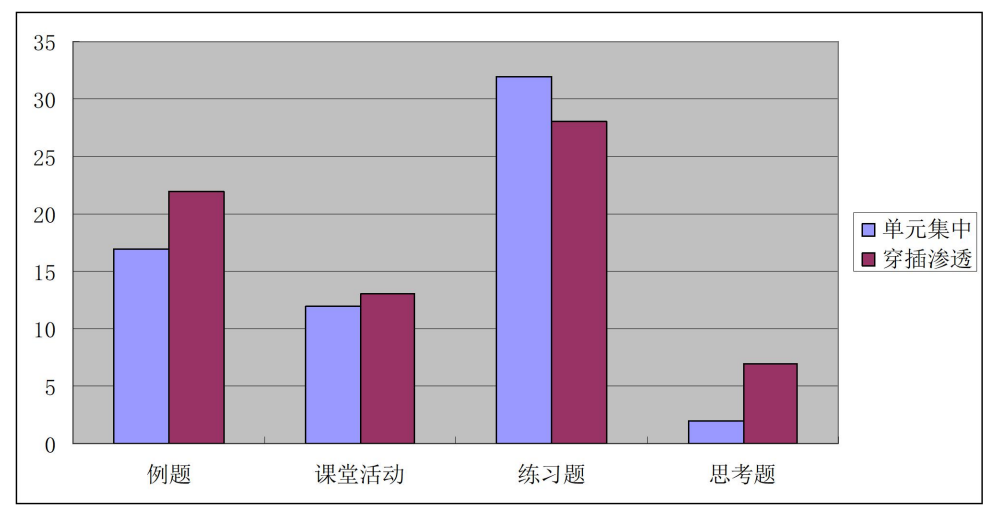

图 1 单元集中与穿插渗透编写问题统计

\section{2 内容设置多样}

作为描述事物本质联系的规律，总是隐藏在具有一定外显形式的事物背后。教材中规律的 外显表现形式主要包括数的规律、式的规律、形的规律以及数与形结合的规律。[1]从探索规 律问题在教材中出现的方式来看，又可分为例题、课堂活动、练习题、思考题、复习题以及综 合与实践等其它方式。我们对西师版小学数学教材探索规律内容的外显形式和出现方式进行了 统计, 结果如图 2 和图 3 所示。

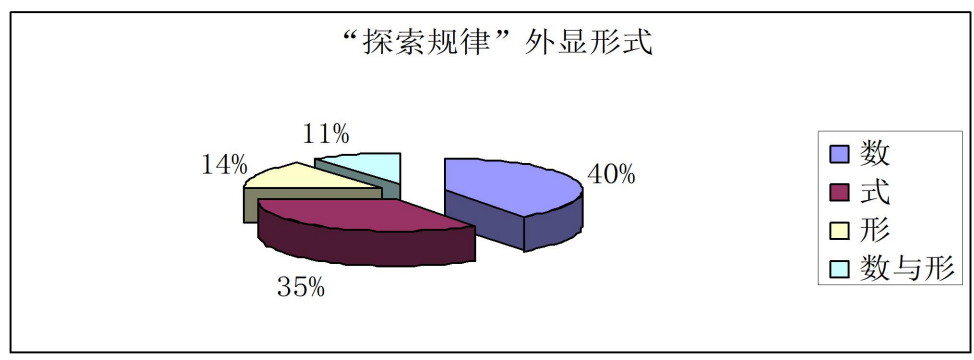

图 2 “探索规律” 的外显形式 


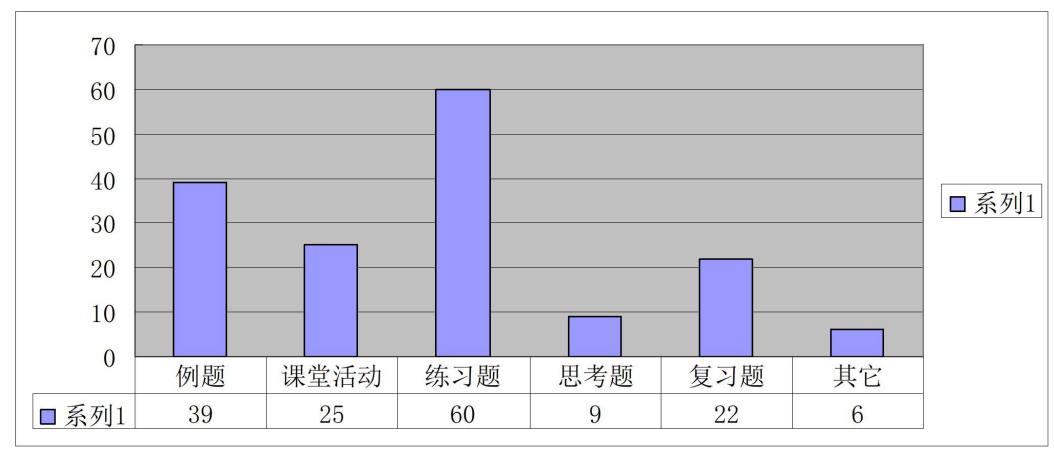

图 3 “探索规律”问题的出现方式

从统计结果看，该套教材中“探索规律”问题的外显形式涵盖了数的规律、式的规律、形的 规律、数与形结合的规律四大类型, 从出现的频率来看, 数的排列规律、式的运算规律出现次 数最多, 符合第一学段和第二学段儿童的认知特点, 也充分考虑了《标准》要求的“素材的选 取要体现课程内容的实质”要求。从“探索规律”问题的现方式多样，包括了例题、课堂活动、 练习题、思考题、整理与复习等, 其中又主要以练习题、例题、课堂活动和复习题的形式出现。 这样编写为实现探索规律的教学价值和目标提供了充分的素材和线索依据。教师在教学中应针 对不同年级学生的认知水平和生活经验, 创造性地使用教材, 对教材中探索规律教学内容从形 式、类型以及数量等多方面进行设计和加工，真正实现探索规律内容的教学价值。

\section{3 体现学生探索规律的过程}

教材中的以独立单元形式编写“探索规律”内容, 设计上非常注重学生探索规律的过程。呈现 方式上以例题, 课堂活动, 练习题的先后顺序呈现本单元的内容。在例题和课堂活动部分, 教 材大量采用“云图”提示的形式，通过设计启发性的提问，对话旁白等来引导学生观察、回忆、 比较、验证、分析等思维过程, 实现经历规律的探索过程, 积累探索规律问题解决的活动经验。

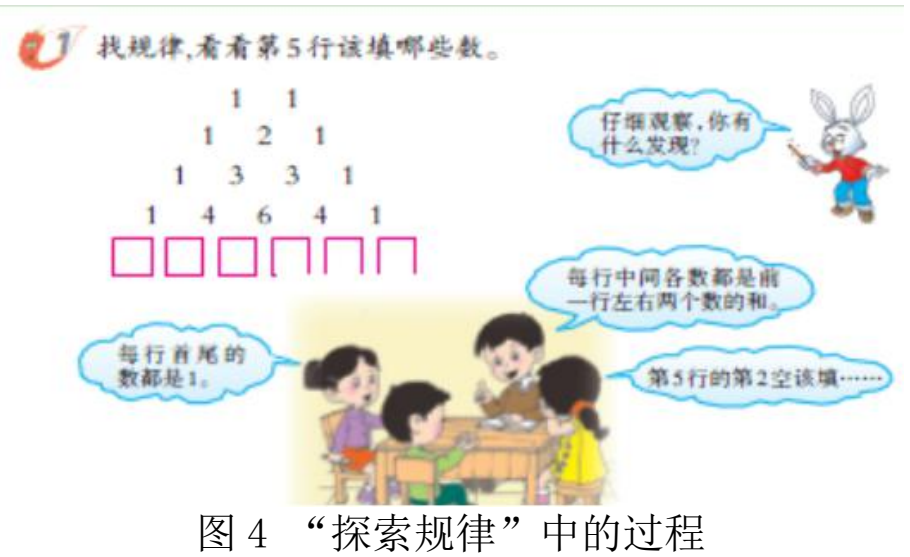

如图 4 教材截图所示。在练习部分则要求学生在尝试中独立探索规律，解决问题，学生 在练习和作业中进一步体验探索规律的完整过程。教材编写上为实现“探索规律”的教学价值创 造了充分的认知和学习空间, 我们在教学中更应该遵循这一思路, 通过创设形式多样、层次分 明的学生活动情境, 让学生经历探索规律问题的解决过程, 充分将《标准》中”过程与方法” 目标落到实处。

\section{4 问题设置注重开放性和创造性}

《标准》提出“创新意识的培养是现代数学教育的基本任务, 应体现在数学教与学的过程 之中”。而开放性的问题是培养学生创新意识的重要载体, 在教材中设置具有开放性的问题可 以对教师教学起到很好的引导作用。在“探索规律”问题的选取中，该套教材注意了问题的开放 
性, 例如像图 5 所示的思考题, 学生可以站在不同的角度给出很多答案, 如从循环规律角度可 为 $10,20, \underline{10}, \underline{20}, \underline{10}, \underline{20}$; 从等差递增的角度可为 $10,20, \underline{30}, \underline{40}, \underline{50}, \underline{60}$; 从倍数递增的角度可为 $10,20, \underline{40}, \underline{80}, \underline{160}, \underline{320}$; 从前两项决定后一项的角度可为 $10,20, \underline{30}, \underline{50}, \underline{80}, \underline{130}, \underline{210}$ 等等。除此之外 该教材在探索规律任务设计上注重让学生创造规律, 例如图 6 所示的问题, 除了要求学生延续 规律外, 还给学生创造规律提供了空间。选取具有一定开放性的问题作为“探索规律”的材料, 既有利于学生从多个角度参与, 体验学习的乐趣与成就, 也有利于激发学生的创造热情, 培养 其创新意识。但从整套教材来看, 这样的问题或情境数量上还比较少, 我们在教学实践中可以 根据学生的实际情况有意识地增强这一部分内容, 让学生体验创造规律的过程, 培养其创新意 识。

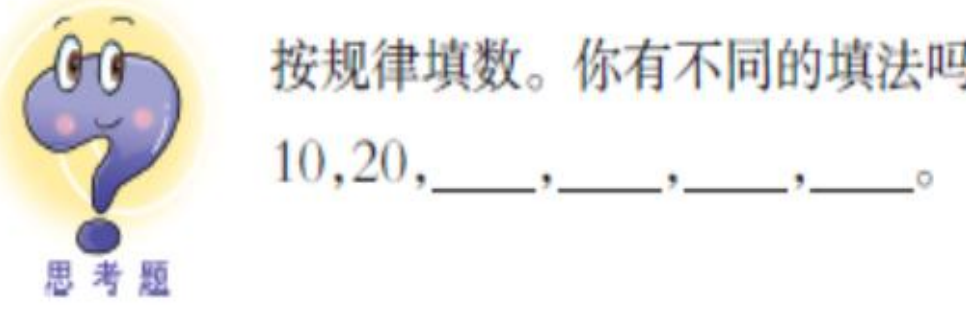

图 5 “探索规律” 中的提问形式

\section{4. 按下面的规律, 用学具卡片摆一摆。}

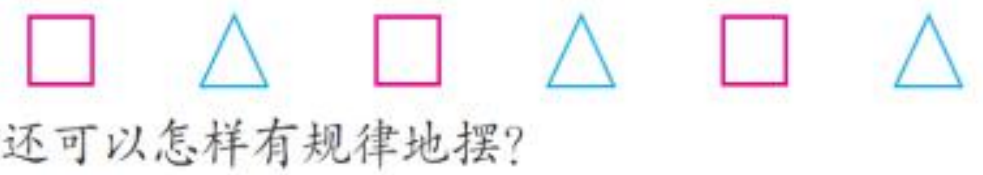

图 6 教材中的探索规律问题

\section{4. 结论与建议}

\section{1 研究结论}

按照《义务教育数学课程标准》要求, 新课标小学数学教材将“探索规律”作为单设内容凸 显了它在数学中的价值和地位, 我们在教学中应基于《标准》的精神和要求, 站在一个较高的 角度理解到“探索规律”问题的实质, 认识到它的教学价值, 准确定位这部分内容的教学要求, 不随意拔高，也不简单处理。

西师版小学数学教材在“探索规律”内容的编写上具有较为鲜明的特色，如在编写模式上既 很好的继承了《大纲》教材穿插、渗透编写的经验, 又以独立单元形式进行集中编写, 体现《标 准》新要求。又如在独立单元编写体例上体现了“规范与统一”, 即按照例题、课堂活动、练习 题的先后顺序编写，和整套教材具有统一的体例。再如在内容的选取上做到“多样与开放”,即 选择了外显形式多样的、具有开放性的规律性问题作为探索的对象等。我们在利用教材进行教 学时, 应基于《标准》, 整体把握教材对“探索规律”内容的编写特点, 领会教材编写者的意图, 并创造性地使用教材, 实现“探索规律”内容的教学价值和目标。

\section{2 教学建议}

\subsection{1 突出过程性和情感教学目标}

教学目标是教学活动的出发点, 也是归宿点。“探索规律”内容的学习并不以知识点或具体 的技能掌握的多少为主要的教学目标, 也不是对前面所学相关知识的综合应用过程。探索规律 学习, 重在一个“探”字, 教师要引导学生在观察中发现, 在比较中猜测, 在验证中修正和确认, 
在应用中建构。因此, 探索规律内容的教学目标要突出过程和情感目标, 把学生体验探索规律 问题解决的过程, 学习观察, 比较, 概括等方法, 积累探索规律问题解决的相关经验, 丰富学 生对数学的认识和领悟，锻造学生完善的人格等确定为探索规律内容教学的主要目标。

\subsection{2 给学生探索留下充足的时间和空间}

为了实现教学目标, 在教学过程设计中, 教师要为学生的探索过程留下足够的时间和空间。 首先教师不要用自己的想法代替学生的思考，而应和学生做“换位思考”，努力尝试站在学生角 度思考探索规律问题的解决过程。其次教师要创设多种层次的学生活动, 发挥小组合作学习的 优势, 让学生动脑想, 开口说, 动手做, 真正把学生学习的主体地位落到实处, 给学生留下足 够的探索、体验过程。

\subsection{3 突出过程性学习评价}

学习评价对学生学习起着导向的作用。探索规律内容的学习, 教师应突出过程性评价, 即 不以知识掌握的多少论英雄, 不以规律问题解决的多少论成败, 而应关注学生在学习过程中是 否真正参与和体验了探索规律的过程, 是否在探索的过程中对数学的思想和方法有所感悟并获 得了积极的情感体验等。

\section{基金项目}

四川省教育厅人文社科一般项目——核心素养视阈下职前中学数学教师教学设计能力的构成 与培养模式研究 (18SB0225)。

\section{参考文献}

[1] C.B.Tang, H.Shao. A teaching field that highlights the essence of Mathematics: "exploration rule", lesson preparation and case analysis. [J]. people's education, 2006, (13):40 - 45..

[2] H.Zhang. Analysis and Reflection on the "law of exploration" in the textbook of primary school mathematics [J]. composition and examination (primary Edition), 2010 (1): 4 - 12.

[3] J.C.Liu, H.Wang. The characteristics of the "law of exploration" in primary school mathematics textbook. [J]. modern primary and secondary education, 2010, (12):15 - 19.

[4] People's Republic of China Ministry of education. Full time compulsory education mathematics curriculum standard (2011 Edition) [S]. Beijing: Beijing Normal University press, January 2012 First Edition: 72 - 73.

[5] N.Q.Song. Compulsory education curriculum standard experimental textbook (Primary Mathematics) [M]. Chongqing: Southwestern Normal University press, June 2012 first edition.

[6] N.Q.Song, D.Z.Zhang. Primary school mathematical education Conspectus [M]. Beijing: Higher Education Press, 2008. 